\title{
Surface Tension Confined (STC) Tracks for Capillary-Driven Transport of Low Surface Tension Liquids
}

Thomas M. Schutzius*

\author{
Mohamed Elsharkawy*
}

Constantine M. Megaridis $*$ $\ddagger$
Manish K. Tiwari ${ }^{\dagger}$

\begin{abstract}
Surface tension confined (STC) open tracks for pumpless transport of low-surface tension liquids (e.g., acetone, ethanol, hexadecane) on microfluidic chips are fabricated using a large-area, wet-processing technique. Wettable, paraffin-wax, submillimeter-wide tracks are applied by a fountain-pen procedure on superoleophobic, fluoroacryliccarbon nanofiber (CNF) composite coatings. The fabricated anisotropic wetting patterns confine the low-surfacetension liquids onto the flow tracks, driving them with meniscus velocities up to $3.1 \mathrm{~cm} \mathrm{~s}^{-1}$. Scaling arguments and Washburn's equation provide estimates of the liquid velocities measured in the STC tracks. These tracks are also shown to act as rails for directional sliding control of mm-sized water droplets. The present facile top-down patterned wettability approach can be extended to deposit micrometer-wide tracks, which bear promise for pumpless handling of low-surface tension liquids (e.g., aqueous solutions containing alcohols or surfactants) in lab-on-a-chip type applications or in low power, high-throughput bio-microfluidics for health care applications.
\end{abstract}

\section{Introduction}

Surfaces and coatings with patterned wettability (e.g., hydrophobic, hydrophilic) have shown potential applications in microfluidics, ${ }^{1}$ fog capture ${ }^{2}$ and pool boiling. ${ }^{3}$ With recent advancements, surfaces with adjacent superhydrophobic and superhydrophilic regions are feasible at a reasonable cost; with properly designed patterns, one can produce microfluidic paths where water is confined and transported by surface tension alone (i.e., surface tension confined tracks). ${ }^{4-6}$ The surface tension $\left(\gamma_{\mathrm{LG}}\right)$ of water is relatively high $\left(\approx 72 \mathrm{mN} \mathrm{m}^{-1}\right)$ as compared with oils $\left(\approx 25 \mathrm{mN} \mathrm{m}^{-1}\right)$ and organic solvents $\left(\approx 20 \mathrm{mN} \mathrm{m}^{-1}\right)$. This makes the design of STC channels for oils and organic solvents more difficult. Zimmermann et al. ${ }^{7}$ demonstrated an excellent route to attaining spatial domains of superoleophobicity and superoleophilicity; however, the apparent CA value for hexadecane $\left(\theta_{\mathrm{C}_{16} \mathrm{H}_{34}}^{*}\right)$ on the oleophobic domain was $\sim 140^{\circ}$ (not quite in the super-repellent regime), with no report of liquid sliding behavior or STC channel velocities, and no

\footnotetext{
${ }^{*}$ Department of Mechanical and Industrial Engineering, University of Illinois at Chicago, Chicago, IL 60607, USA

${ }^{\dagger}$ Laboratory of Thermodynamics in Emerging Technologies, Mechanical and Process Engineering Department, ETH Zurich, 8092 Zurich, Switzerland

‡cmm@uic.edu
} 
wettability mention for alcohols. In one application, Chang et al. ${ }^{8}$ used oleophobic/oleophilic patterning to produce microchips by a capillary-driven, self-assembly process. In another publication, Kobaku et al., ${ }^{9}$ fabricated site-selective, self-assembling surfaces for low-surface tension liquids; the patterned superomniphobic-superomniphilic surfaces targeted applications ranging from surface directed microchannels for liquid phase reactions, to site-selective condensation of heptane vapors, and preferential nucleation of methanol under boiling conditions. Such self-assembling surfaces also can enable low-cost patterning of solution-processed polymers or suspensions of micro/nanoparticles. It should be noted that the focus of that work was on site-specific self-assembly, while the emphasis of the present work is on facile pumping of low-surface tension liquids and low-cost, large-area fabrication. Despite many attractive application prospects in synthetic biology, chemistry and heat transfer, only limited literature exists today on substrates with spatially-patterned wettability for low surface tension liquids.

We report a facile method to produce large-area, STC tracks for low-surface tension liquids. These 2-D tracks attain liquid velocities approaching $3 \mathrm{~cm} \mathrm{~s}^{-1}$ for low-viscosity liquids, thus bridging a gap in the literature for unassisted, capillary driven, open channel micro-fluidic transport of oils, solvents and alcohols. The STC tracks are fabricated from spray coating a fluoroacrylic-carbon nanofiber dispersion to form a superoleophobic surface, which is subsequently patterned with a parrafin wax-hexane solution to form wettable tracks. To demonstrate the facile nature of the present technique, the wax patterning is done by a continuous fountain-pen printing method-a relatively simple process. However, the technique should be amenable to other finer-scale liquid patterning processes (e.g., ink-jetting, etc.), which can attain spatial resolutions in the micrometer regime. Spontaneous transport of water (which only partially wets the wax) in the tracks is not observed; however, anisotropic wetting behavior is seen, ${ }^{10}$ as mm-size water droplets slide under the influence of gravity along the track direction, but remain pinned when tilted against the non-wettable curbs. ${ }^{11}$ In this case, tracks act to guide sliding water droplets with the aid of gravity.

\section{Experimental}

The dispersion formulation used to form the superoleophobic base coating has been reported elsewhere; ${ }^{12}$ a brief description is given here for completeness. First, carbon nanofibers (CNF), (PR-24-XT-HHT; Pyrograf III) were acid treated with acetic acid (99.9 wt.\%, ACS Certified, Fisher Scientific) and bath sonicated (output power $70 \mathrm{~W}$; frequency $42 \mathrm{kHz}$; Cole-Parmer, Model 08895-04) for 10 min. producing a 'mud-like' material (2.5 wt.\% CNF in acetic acid), which was subsequently diluted with acetone ( $0.9 \mathrm{wt} . \% \mathrm{CNF}$ in acid and acetone) and probe sonicated (750 W, $13 \mathrm{~mm}$ probe dia., $40 \%$ amplitude, $20 \mathrm{kHz}$ frequency, Sonics \& Materials, Inc., Model VCX-750) for 1.5 min. Separately, an aqueous fluoroacrylic copolymer dispersion (PMC, 20 wt.\% in water, Capstone ST-100, DuPont) was dilluted with acetic acid and then acetone to form a 2.9 wt.\% PMC stock solution (1:2.5:5 wt. ratio of water:acetic acid:acetone). This stock solution was then added to the stable CNF-acetone-acetic acid suspension (1:1.5 wt. ratio PMC:CNF), which was then bath sonicated for 10 min until a stable dispersion was formed. The dispersion was subsequently spray cast with an airbrush (Paasche VL siphon feed, $0.78 \mathrm{~mm}$ spray nozzle) onto aluminum plates or glass slides using compressed air (2.1 bar). The coatings were initially dried by a heat-gun (Proheat ${ }^{\circledR}$ Varitemp ${ }^{\circledR}$ PH-1200, $1300 \mathrm{~W}$ 
max) for several minutes and were then placed in an oven at $70^{\circ} \mathrm{C}$ for 60 min to dry completely, thus forming the oleophobic base coating for the subsequent step (wettability patterning). For that, a 1 wt.\% (0.7 vol.\%) solution of parrafin wax (melting point $53-57^{\circ} \mathrm{C}$, Sigma-Aldrich) in hexane ( $>98.5 \%$, ACS reagent, Sigma-Aldrich) was formed by adding wax to hexane and shaking mechanically for several minutes at room temperature. Patterning was done by a continuous fountain-pen printing technique (see Fig. 1) whereby the wax solution was dispensed with a syringe pump (Cole-Parmer, 74900 Series) out of an 18 gauge polypropylene needle (1.27 mm outer dia., $0.84 \mathrm{~mm}$ inner dia., 12.7 mm length; $\left.\mathrm{EFD}^{\circledR}\right)$ at $15 \mu \mathrm{L} \mathrm{m^{-1 }}$; the needle was kept at a fixed distance of $250 \mu \mathrm{m}$ above the coating, while the substrate was translated at a fixed speed $\left(3.7 \mathrm{~mm} \mathrm{~s}^{-1}\right.$; Velmex translation stage). Patterned areas were subsequently dried quickly with the heat-gun. Track widths of $\approx 1 \mathrm{~mm}$ were readily formed under these process parameters. Since the track width is proportional to the needle diameter, features well below $1 \mathrm{~mm}$ were also printed with a 31 gauge needle (0.24 mm outer dia., $0.1 \mathrm{~mm}$ inner dia.). Figure 1 shows a description of the fountain-pen printing setup.

Wettability characterization was done by measuring advancing and receding contact angle (CA) values for uniform wettability areas by the sessile drop method, whereby 5-10 $\mu \mathrm{L}$ of each probe liquid was dispensed (advancing measurement) and removed (receding measurement). Advancing and receding CAs were captured with a high-speed, backlit image acquisition setup (Redlake MotionPro); a different spot location on the coated substrate was used for each measurement. The morphology of the unpatterned and patterned, spray deposited coating was characterized by scanning electron microscopy (SEM) analysis (Hitachi S-3000 N). All samples were coated with a conforming $4 \mathrm{~nm}$ layer of Pt/Pd prior to SEM imaging. Liquid transport along the patterned STC tracks was captured with a high-speed camera (frame rate $50 \mathrm{~s}^{-1}$, Redlake MotionPro) and was used to measure flow velocity. For each experiment, a droplet of the appropriate fluid ( $3.7 \mu \mathrm{L}$ acetone; $3.8 \mu \mathrm{L}$ ethanol; $4.5 \mu \mathrm{L}$ hexadecane) was placed at one end of a $1 \mathrm{~mm} \times 25$ $\mathrm{mm}$ track and the impending flow was vizualized at high speed from above.

\section{Results and Discussion}

Over the past few years, many studies have investigated liquid repellent surfaces generated from spray deposited polymer-particle dispersions; the polymer acts to reduce surface energy and the role of the filler is to create surface texture. ${ }^{13,14}$ In the present coatings, the overlapping CNF particles create inherently re-entrant textures (see Fig. 2), which are necessary for superoleophobicity $\left(\theta_{\mathrm{A}}^{*}>150^{\circ}\right.$; see Table 1$) .{ }^{15}$ Table 1 shows that the self-cleaning behavior of the coating (i.e., low CA hysteresis) is lost with lower surface tension liquids, such as alcohols. However, $\theta_{\mathrm{A}}^{*}$ retains values indicating that alcohols do not spread spontaneously on these coatings. For the case of ethanol, $\theta_{\mathrm{A}}^{*}$ is relatively high, implying a non-trivial differential pressure across the droplet interface (i.e., Laplace pressure). Recently, high Laplace pressures inside water droplets interacting with superhydrophobic surfaces have been shown to be useful for self-pumping, superhydrophobic-patterned surface microfluidic $\left(\mathrm{S}^{2} \mathrm{M}\right)$ platforms, where liquid motion is induced by the merging of a larger droplet with a smaller droplet, both of which are connected by a microchannel. ${ }^{16}$ For water, contact angle hysteresis has been shown to be important in two-dimensional lab-on-paper devices where water contact angle hysteresis is selectively increased in predefined areas on a superhydrophobic paper-so called hysteresis enabled 
lab-on-paper (HELP); such an approach enables one to perform storage, guided transport, mixing and sampling of test liquids. ${ }^{17}$ Such clever techniques can be extended to low-surface tension liquids only if the surfaces contain wettability patterns capable of confining low-surface tension fluids, which is non-trivial to achieve. Our facile approach to form such patterns uses an approach that circumvents sophisticated top-down microfabrication steps. In addition, our technique-wherein we apply wax solution locally to selectively increase the surface energy on predefined tracksresults in considerable materials saving. We focus on pumpless transport of low-surface tension liquids in open, surface-tension-confined tracks by capillary action-a different mechanism than in the $\mathrm{S}^{2} \mathrm{M}$ and HELP devices, which are mentioned here only to show the wide variety of potential applications of the present approach.

On smooth surfaces with high surface energy, droplets may occupy one of two possible wetting states: 1) total wetting, or 2) partial wetting. The specific wettability state can be predicted using the spreading parameter $S$, defined as

$$
S=\gamma_{\mathrm{SG}}-\left(\gamma_{\mathrm{SL}}+\gamma_{\mathrm{LG}}\right)
$$

where $\gamma_{\mathrm{SG}}$ and $\gamma_{\mathrm{SL}}$ denote the surface tensions of the solid/gas and solid/liquid interfaces, respectively. The critical surface tension of the solid, which is denoted by $\gamma_{\mathrm{C}}$, corresponds to $S=0 . S>0$ (i.e., $\gamma_{\mathrm{LG}}<\gamma_{\mathrm{C}}$ ) predicts total wetting (i.e., equilibrium CA $\theta_{\mathrm{E}}=0$ ), while $S<0$ corresponds to partial wetting (i.e., $\theta_{\mathrm{E}} \neq 0$ ). $S=0$ marks the transition between the two wetting states. For liquids satisfying the condition $S>0$, spontaneous transport in STC channels is expected a priori; however, for liquids with $S<0$, spontaneous liquid spreading is not guaranteed. The above discussion applies for smooth surfaces. For rough surfaces, partial wetting may give rise to a situation referred to as hemi-wicking, ${ }^{18}$ where the liquid invades the texture but leaves exposed a portion of the solid. This configuration is thermodynamically favored when $\theta_{\mathrm{E}}$ satisfies the inequality

$$
\theta_{\mathrm{E}}<\theta_{\mathrm{c}} \text { with } \cos \theta_{\mathrm{c}}=\frac{1-\Phi_{\mathrm{S}}}{r-\Phi_{\mathrm{S}}}
$$

where $\Phi_{\mathrm{S}}\left(\Phi_{\mathrm{S}}<1\right)$ denotes the unwetted fraction of solid (1- $\Phi_{\mathrm{S}}$ is then the wetted fraction of the solid), and $r$ is the roughness of the solid ( $r \geq 1$, ratio of true surface area to projected area). ${ }^{19}$ Estimates of equilibrium CA on parrafin wax $\left(\theta_{\mathrm{E}, \text { wax }}\right)$ for several of the probe liquids utilized in this study can be found in Fox \& Zisman; ${ }^{20}$ in general, $\gamma_{\mathrm{C}}$ for the $\mathrm{CH}_{3}$ and $\mathrm{CH}_{2}$ bonds (i.e., parrafin wax) is in the range of $22-31 \mathrm{mN} \mathrm{m}^{-1}$, ${ }^{21}$ which overlaps with the surface tensions of the organic probe liquids investigated herein (see Table 1). Considering the case where $\Phi_{\mathrm{S}}<<1$ (i.e., large area fraction of liquid in channels), Eq. 2 becomes

$$
\cos \theta_{\mathrm{c}} \approx \frac{1}{r}
$$

When $\gamma_{C}$ and $\gamma_{\mathrm{LG}}$ are comparable - as is the case here-then from Young's relation ${ }^{19}$ it follows that $\theta_{\mathrm{E}}$ should be relatively low; for hexadecane ${ }^{20} \theta_{\mathrm{E} \text {, wax }}=27^{\circ}$. Setting $\theta_{\mathrm{E}, \text { wax }}=\theta_{\mathrm{c}}=27^{\circ}$ and substituting into Eq. 3 , it follows that $r=1.06$, implying that for the case of $S<0$ (partial wetting), only a relatively small amount of surface roughness is required to produce hemi-wicking behavior. This level of roughness is well below that achieved by spray cast coatings 
which contain high concentrations of filler particles (cf. Fig. 2, 60 wt.\% CNF). So if the wax were deposited as a conformal, thin layer onto the highly textured coating, then it would be possible to produce hemi-wicking behavior. The wax should be solution-processed with a low-surface tension liquid so as to partially wet $(S<0)$ the underlying textured surface, and ultimately, produce patterns on the highly liquid-repellent coating. From Table 1 we deduce that hexane does not fully wet the CNF coating, so this liquid offers an appropriate choice for patterning wettable tracks on top of the superhydrophobic terrain; fully wetting liquids, which spread uncontrollably, would make it extremely difficult to pattern with good resolution. When applying the wax coating, it is necessary to preserve the underlying surface texture, otherwise the desired hemi-wicking behavior may not be attained. The deposited wax thickness is a function of printing speed, wax-hexane solution flow rate, the printing nozzle diameter and solution concentration. From Fig. 2, the surface texture appears to have four apparent length scales: 1) micro-clusters of CNFs (see a; with length scale $\approx 10 \mu \mathrm{m}), 2) \mathrm{CNF}$ diameter (see $\left.\left.\mathbf{b} ; \approx 100 \mathrm{~nm}^{12}\right), 3\right) \mathrm{CNF}$ length $\left(\approx 10-30 \mu \mathrm{m}^{12}\right)$ and 4$)$ pores in-between adjacent CNFs (see $\mathbf{b} ; \approx 1 \mu \mathrm{m}$ ). If one wishes to preserve coating feature sizes larger than $\approx 1 \mu \mathrm{m}$ (i.e., the large micro-clusters seen in Fig. 2), then one must ensure that the wax film thickness is approximately the same or below this value. The substrate translation speed ( $v$ ) and dispensing volumetric flow rate $(Q)$ are related through $Q \sim v h_{\text {film }} d$, where $d$ is the inner diameter of the nozzle and $h_{\text {film }}$ is the film thickness of the deposited wax-hexane solution. The relation between the film thickness of the wax-hexane solution and the dry wax coating thickness $\left(h_{\mathrm{wax}}\right)$ is $h_{\mathrm{wax}}=c h_{\mathrm{film}}$, where $c$ is the volumetric concentration of wax in hexane. Taking $Q=15 \mu \mathrm{L} \mathrm{min}{ }^{-1}, h_{\text {film }} \approx 100 \mu \mathrm{m}$ and $d=0.84 \mathrm{~mm}, v$ becomes $3.0 \mathrm{~mm} \mathrm{~s}^{-1}$. Our substrate velocity in the track deposition experiments was $3.7 \mathrm{~mm} \mathrm{~s}^{-1}$, which is comparable to the calculated value. We applied three successive layers of 0.7 vol. $\%$ solution on each area to produce the desired wetting behavior (i.e., capillary driven flow), which implies an overall wax-layer thickness of $\approx 2 \mu \mathrm{m}$. Thicker, as well as thinner, wax layers resulted in channels with unfavorable capillary behavior (i.e., no liquid movement). Figure 3 presents SEM micrographs of a wax-patterned, composite coating. We see that most of the low-lying valleys have been filled, but the micro-cluster texture remains in the areas protruding above the polymer. The space between adjacent micro-clusters forms a channel, as is evident from Fig. 3b. The average micro-cluster interspacing was estimated to be $18 \pm 7 \mu \mathrm{m}$, as based on 66 distinct measurements.

An important property of microchannels is the fluid advancing velocity. For our STC tracks, the dynamic transport is affected by both viscous and capillary forces. ${ }^{22}$ We assess the moving liquid front's velocity by modeling the liquid advancement as capillary driven impregnation of the liquid into the pores of the rough underlying surface. The pores can be imagined as small channels in their own right. A balance of capillary and viscous forces yields ${ }^{23}$

$$
u \sim \frac{\Delta p d_{\text {pore }}^{2}}{\mu x} \sim \frac{\Delta \gamma d_{\text {pore }}}{\mu x}
$$

where $\Delta p \sim \Delta \gamma / d_{\text {pore }}$ represents the Laplace pressure due to the presence of a curved liquid-gas interface (in between the protrusions seen in Figure $3 b$ ), $d_{\text {pore }}$ is the average pore diameter (selected values depicted by the arrows in Figure 3b), $\mu$ the dynamic viscosity of the fluid and $x$ the position of the advancing liquid front, which varies with time; $\Delta \gamma=\gamma_{\mathrm{SG}}-\gamma_{\mathrm{SL}}$ describes the surface energy change as the fluid column advances. For highly wetting cases (i.e., $\left.\theta_{\mathrm{E}} \approx 0\right)$ where $\gamma_{\mathrm{SG}}>>\gamma_{\mathrm{SL}}\left(\right.$ e.g., for hexadecane), ${ }^{20}$ from Young's relation ${ }^{19} \gamma_{\mathrm{SG}} \approx \gamma_{\mathrm{LG}}$, and we deduce $\Delta \gamma \approx \gamma_{\mathrm{LG}}$. 
Stated simply, for highly wetting situations $\Delta \gamma$ can be approximated by the surface tension of the liquid. Table 2 presents the measured velocity $\left(\frac{\Delta x}{\Delta t}\right)$ of the advancing liquid front for the first $1.0 \mathrm{~cm}$ of travel, as well as the values computed from Eq. 4 for $d_{\text {pore }}=18 \mu \mathrm{m}$ (the previously measured average pore scale). The order of magnitude agreement between the predictions and the measurements is quite good, although we have neglected the energy cost of generating new free surface as the liquid flows along the STC tracks and through the obstacles posed by the CNF protruding clusters.

The data listed in Table 2 shows that Equation 4 overestimates the measured velocity more for the case of acetone and ethanol, as compared with hexadecane. The reason for this disrecpency may be due to the approximation of $\Delta \gamma$. In the case of alkanes, it is known that the magnitude of the previously neglected term, $\gamma_{\mathrm{SL}}$, is less than in the case of oxygen-containing liquids. ${ }^{20}$ Considering that $\theta_{\mathrm{E}, \text { wax }}=33 \pm 2^{\circ}$ for ethanol ${ }^{24}$ and $27^{\circ}$ for hexadecane, ${ }^{20}$ from Young's relation the respective values for $\Delta \gamma$ are 18.4 and $24.1 \mathrm{mN} \mathrm{m}^{-1}$. The error of the approximation $\Delta \gamma \approx \gamma_{\mathrm{LG}}$ is then $19 \%$ and $12 \%$, respectively for ethanol and hexadecane. Therefore, for liquids containing oxygen (such as ethanol), one should exercise reserve in applying this approximation. On the other hand, for highly volatile liquids like acetone, this approximation may be the only possible approach due to difficulties associated with measuring $\theta_{\mathrm{E}}$ on wax (rapidly changing liquid volume due to evaporation). In this case, one should expect that $\Delta \gamma$, and ultimately $u$, would be overestimated (as seen in Table 2).

Figure 4 draws the measured values of the liquid wetting front position along an STC track as a function of time for liquids of different viscosity and comparable surface tension (acetone, ethanol, hexadecane; see Fig. 5 for an image sequence of hexadecane transport on a straight track). As expected, increasing viscosity (from acetone to ethanol to hexadecane) results in slower liquid velocities on the respective track. In order to better describe the wetting behavior (i.e., liquid penetration), we utilize the familiar Washburn equation ${ }^{22}$

$$
x=\sqrt{\frac{\gamma_{\mathrm{LG}} d_{\mathrm{pore}} t}{4 \mu}}
$$

which is also plotted in Fig. 4 for acetone, ethanol and hexadecane with $d_{\text {pore }}=18 \mu \mathrm{m}$. The trends are quite consistent, although it appears that Eq. 5 significantly underestimates the advancing wetting front for hexadecane, as compared with acetone and ethanol. This is a complex issue and at its core demonstrates the limitations of the applicability of Washburn's equation to the present data sets. First, Washburn's equation neglects the generation of new free surface (costly in terms of energy), which should ultimately slow the velocity of the advancing liquid. For the idealized case of wetting of pillars-where the tops of the pillars are also wet (i.e., $\theta_{\mathrm{E}} \approx 0$ ) - the percentage of surface energy consumed by generating a new free surface as a liquid advances is $1 /(1+r) .{ }^{19}$ Even for a highly rough case with $r=3,{ }^{18}$ the relative amount of surface energy consumed by free surface generation is $25 \%$. Lower values of $r$ would raise this estimate. Ultimately most important is the effective pore size or characteristic length scale. It is tempting to idealize a textured surface as a series of hollow cylinders due to the ease of which one can model fluid transport in between such cylinders. While we performed SEM and measured characteristic length scales that we felt were representative of an effective micro-channel width (idealized as a pore in Eq. 5), this characteristic length scale had a standard deviation 
of almost $60 \%$ around its mean value, which highlights the large discrepancy from the idealized case. Neveretheless, despite its potential short-comings, Eq. 5 models the wetting behavior for acetone and ethanol quite well.

Patterning the wettability of surfaces is likely to have a wide palette of applications that vary depending on the surface tension of the liquid being used. As an example, the method could be employed for facile fabrication of surfaces with anisotropic wettability. ${ }^{10}$ The following illustrates how the method could be implemented to gain directional movement control of small liquid volumes on a pre-patterned surface. Since we utilized wax in the oleophilic tracks, it is expected that these tracks would be partially-to-fully superhydrophobic. Figure 6(a) is a schematic demonstrating the anisotropic wetting of mm-sized water droplets (blue) inside a mm-wide STC track, where $\alpha$ and $\beta$ define the tilting angles around two orthogonal axes. Figures 6(b)-(c) show the shape of the water droplet placed on a line STC track in an untilted $\left(\alpha=0^{\circ}\right)$ and tilted $\left(\alpha=90^{\circ}\right)$ state, respectively (b-c camera view fixed). In $\mathbf{c}$, note the non-circular solidliquid contact area of the water droplet, which remains immobile and pinned against the hydrophobic curbs. Figures 6(d)-(f) show an image sequence of a water droplet on a track in an untilted and tilted state; the droplet demonstrates its mobility in Figs. 6(e)-(f). The required minimum angle $\beta$ for droplet sliding was $25 \pm 4^{\circ}$-an increased value over unpatterned (superhydrophobic) areas of the coating where droplets slid at tilt angles below $10^{\circ}$. The anisotropic wetting property can be exploited in these open tracks to guide large water droplets with the aid of gravity. The sine of the minimum sliding angle of a droplet with an idealized rectangular solid/liquid contact area can be calculated as $\left(w \gamma_{\mathrm{LV}}\left(\cos \theta_{\mathrm{R}}^{*}-\cos \theta_{\mathrm{A}}^{*}\right)\right) /(\rho V g)$, where $w$ is the width of the wettable track, $\rho$ the fluid density, $V$ the droplet volume, and $g$ the acceleration of gravity. ${ }^{25,26}$ From the above expression, we see that by reducing $w$-the patterned track width-we can reduce the overall sliding angle of the droplet, i.e., increase the mobility of the droplet on the track.

\section{Conclusion}

In summary, we demonstrated the ability to fabricate a surface microfluidic device featuring surface tension confined tracks for pumpless transport of low-surface tension liquids using large-area, scalable techniques—namely spray and continuous fountain pen printing. Low-surface tension liquids, such as acetone, ethanol and hexadecane, attained advancing meniscus velocities up to $3.1 \mathrm{~cm} \mathrm{~s}^{-1}$ on said tracks. The meniscus advancement along the tracks was characterized by combining high speed imaging and a scaling analysis of capillary-viscous force balance for liquid impregnation into the surface texture-similar to Washburn's theory. In a separate application, the tracks were used to allow selective and directionally controlled transport of water droplets in a prefered sliding direction with the aid of gravity. In general, such patterns can find application in low-cost, scalable technologies for lab-on-a-chip and other surface microfluidic devices designed to selectively confine and deliver liquids with a wide-range of surface tensions without the need for pumps. Several other existing surface microfluidic platforms envisioned to benefit from this approach were also presented; currently they are used for water, but with the present approach-and possible modification of the patterning polymer-implementation to low-surface tension liquids may be possible. 


\section{References}

[1] P. Lam, K. J. Wynne and G. E. Wnek, Langmuir, 2002, 18, 948-951.

[2] L. Zhai, M. C. Berg, F. C. Cebeci, Y. Kim, J. M. Milwid, M. F. Rubner and R. E. Cohen, Nano Letters, 2006, 6, $1213-1217$.

[3] A. R. Betz, J. Xu, H. H. Qiu and D. Attinger, Applied Physics Letters, 2010, 97, 141909.

[4] J.-T. Lee, M. C. George, J. S. Moore and P. V. Braun, Journal of the American Chemical Society, 2009, 131, $11294-11295$.

[5] Y. H. Han, P. Levkin, I. Abarientos, H. W. Liu, F. Svec and J. M. J. Frechet, Analytical Chemistry, 2010, 82, $2520-2528$.

[6] D. Zahner, J. Abagat, F. Svec, J. M. J. Frechet and P. A. Levkin, Advanced Materials, 2011, 23, 3030-3034.

[7] J. Zimmermann, M. Rabe, G. R. J. Artus and S. Seeger, Soft Matter, 2008, 4, 450-452.

[8] B. Chang, V. Sariola, S. Aura, R. H. A. Ras, M. Klonner, H. Lipsanen and Q. Zhou, Applied Physics Letters, 2011, 99, 034104.

[9] S. P. R. Kobaku, A. K. Kota, D. H. Lee, J. M. Mabry and A. Tuteja, Angewandte Chemie International Edition, 2012, 51, 10109-10113.

[10] K.-H. Chu, R. Xiao and E. N. Wang, Nature Materials, 2010, 9, 413-417.

[11] H. Mertaniemi, V. Jokinen, L. Sainiemi, S. Franssila, A. Marmur, O. Ikkala and R. H. A. Ras, Advanced Materials, 2011, 23, 2911-2914.

[12] A. Das, T. M. Schutzius, I. S. Bayer and C. M. Megaridis, Carbon, 2012, 50, 1346-1354.

[13] I. S. Bayer, M. K. Tiwari and C. M. Megaridis, Applied Physics Letters, 2008, 93, 173902.

[14] M. K. Tiwari, I. S. Bayer, G. M. Jursich, T. M. Schutzius and C. M. Megaridis, ACS Applied Materials \& Interfaces, 2010, 2, 1114-1119.

[15] A. Tuteja, W. Choi, M. L. Ma, J. M. Mabry, S. A. Mazzella, G. C. Rutledge, G. H. McKinley and R. E. Cohen, Science, 2007, 318, 1618-1622.

[16] S. Xing, R. S. Harake and T. Pan, Lab On A Chip, 2011, 11, 3642-3648.

[17] B. Balu, A. D. Berry, D. W. Hess and V. Breedveld, Lab On A Chip, 2009, 9, 3066-3075.

[18] J. Bico, U. Thiele and D. Quéré, Colloids and Surfaces A: Physicochemical and Engineering Aspects, 2002, 206, $41-46$. 
[19] P. G. de Gennes, F. Brochard-Wyart and D. Quéré, Capillarity and Wetting Phenomena: Drops, Bubbles, Pearls, Waves, Springer, 2004.

[20] H. W. Fox and W. A. Zisman, Journal of Colloid Science, 1952, 7, 428-442.

[21] W. A. Zisman, Advances in Chemistry Series, 1964, 43, 1-51.

[22] E. W. Washburn, The Physical Review, 1921, 17, 273-283.

[23] T. M. Squires and S. R. Quake, Reviews of Modern Physics, 2005, 77, 977-1026.

[24] H. Kamusewitz, W. Possart and D. Paul, Colloids and Surfaces A: Physicochemical and Engineering Aspects, 1999, 156, 271-279.

[25] C. G. L. Furmidge, Journal of Colloid Science, 1962, 17, 309-324.

[26] C. W. Extrand and A. N. Gent, Journal of Colloid and Interface Science, 1990, 138, 431-442.

[27] CRC Handbook of Chemistry and Physics, ed. D. R. Lide, CRC Press, Boca Raton, FL, 85th edn., 2005. 


\section{Figures}

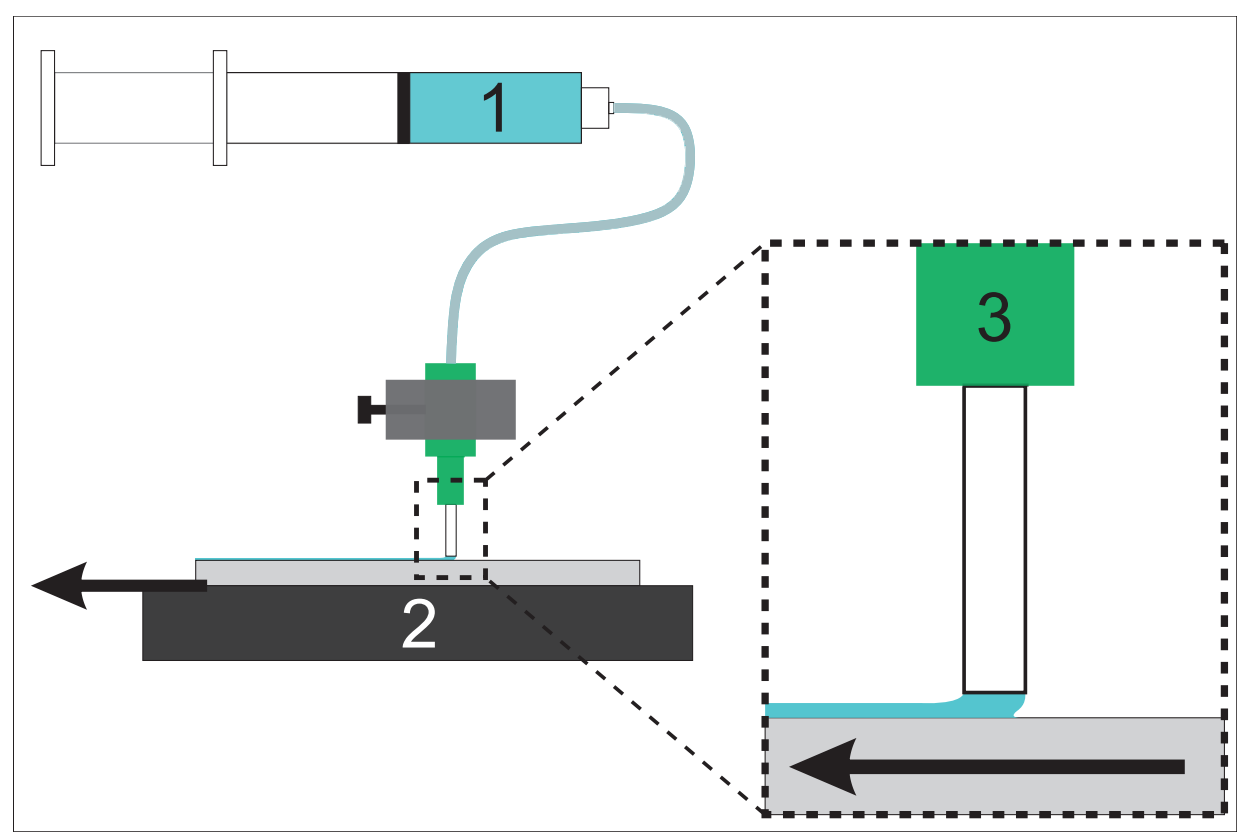

Figure 1: Continuous fountain-pen printing setup: 1) Syringe containing wax-hexane solution (dispensed by an automated syringe pump, not pictured); 2) linearly translating substrate (attained by a computer controlled linear actuator, not pictured); 3) detail showing polypropylene nozzle dispensing wax-hexane solution onto the coated (oleophobized) glass slide, substrate moving right-to-left, nozzle held at a fixed position. 


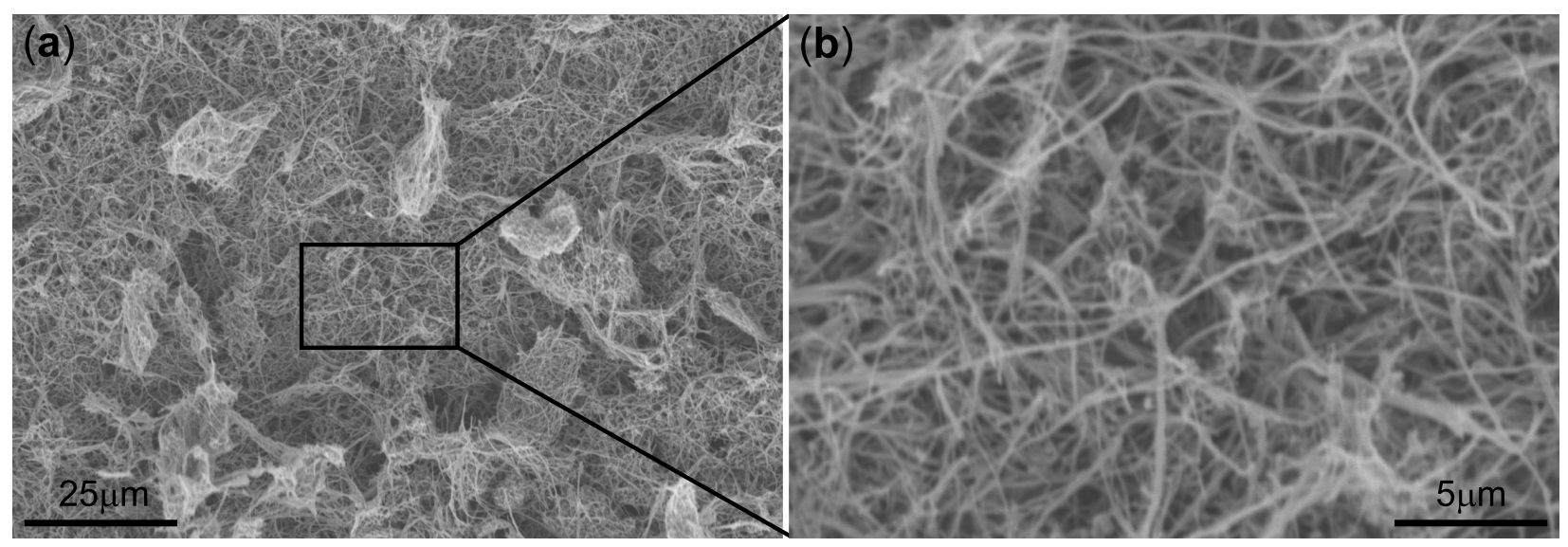

Figure 2: SEM micrographs of a superoleophobic, spray-deposited, fluoroacrylic/CNF composite coating with 60 wt.\% $\mathrm{CNF}$ at (a) low-magnification and (b) high-magnification. 

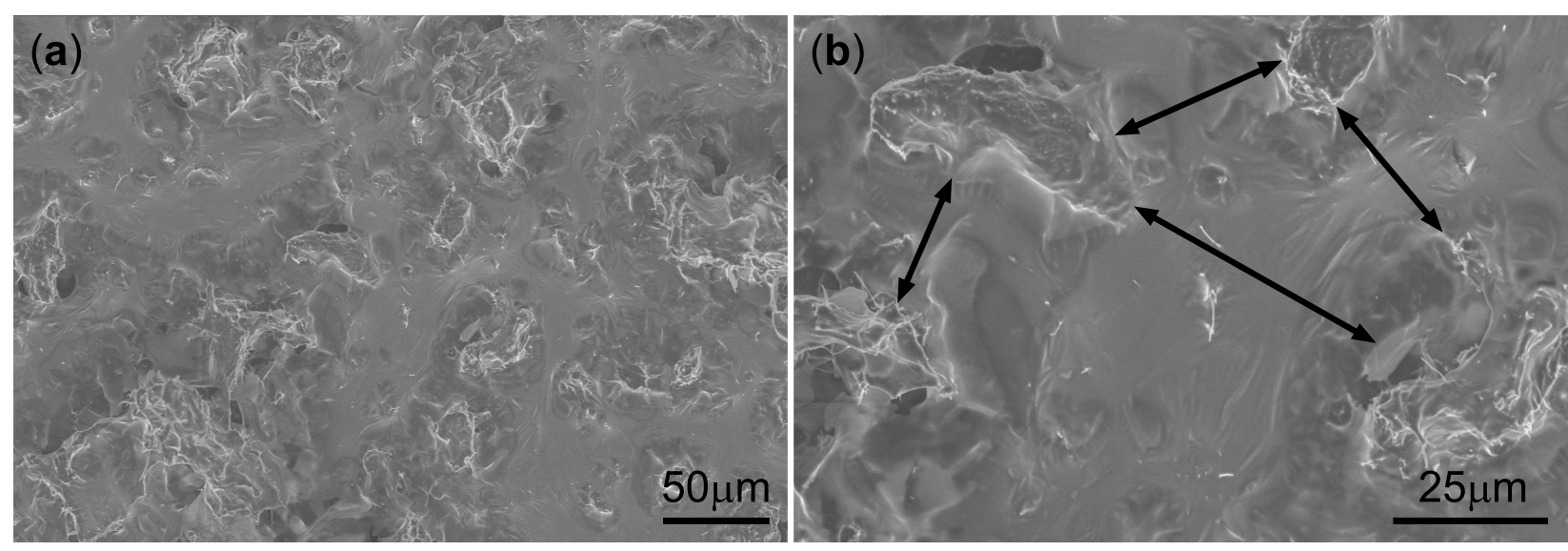

Figure 3: SEM micrographs of a wax-coated, fluoroacrylic/CNF (60 wt.\% CNF) composite coating at (a) lowmagnification and (b) high-magnification. Arrows in (b) demonstrate example measurements of the interspacing values between micro-texture features that remain exposed after wax-patterning. 


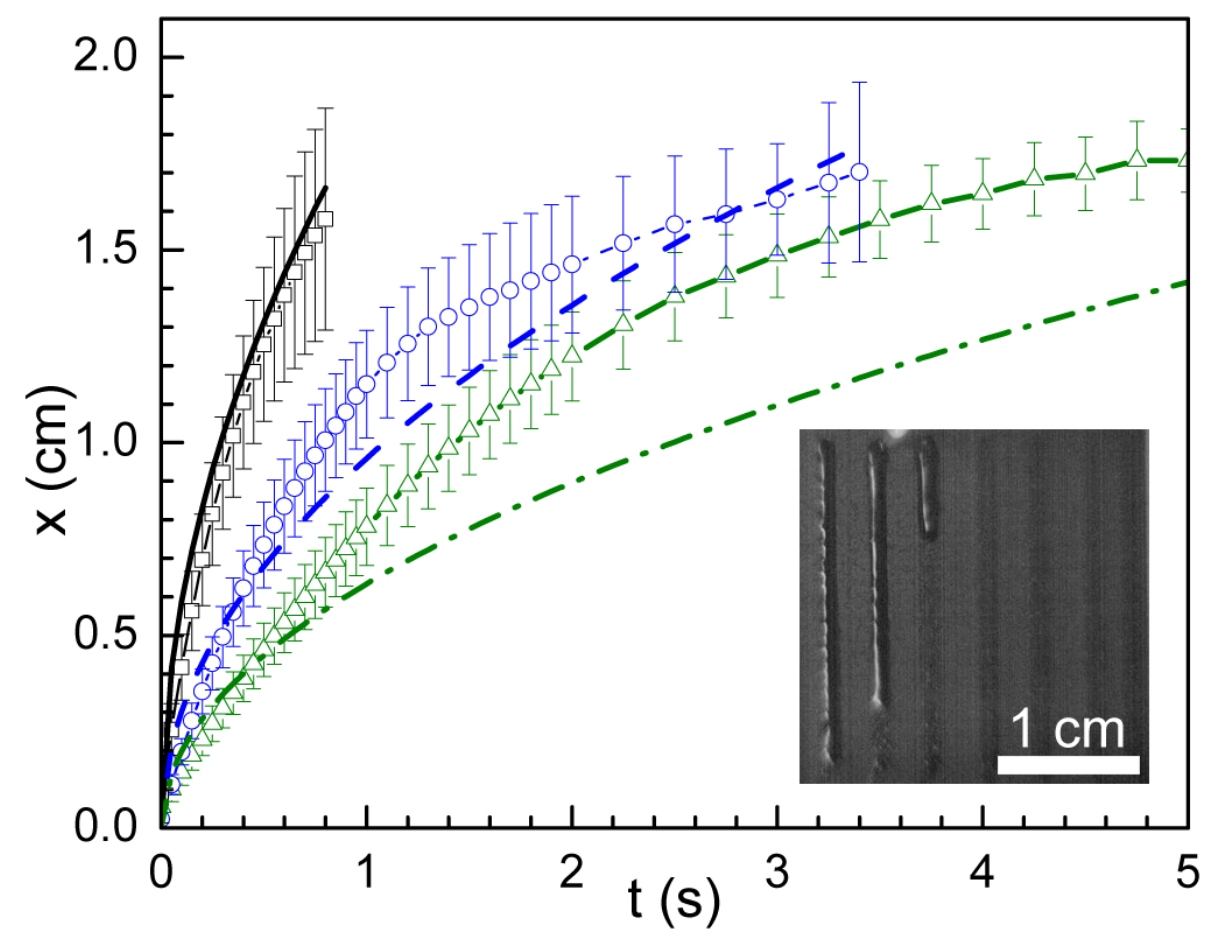

Figure 4: Measured data for liquid wetting meniscus position $(x)$ as a function of time $(t)$ for acetone (一 $\square$; average of 10 trials), ethanol (- - $\bigcirc---$; average of 14 trials) and hexadecane (- $-\triangle \triangle--\_$; average of 14 trials) flowing in wax-patterned STC tracks. Washburn theory ${ }^{22}$ prediction of $x$ vs. $t$ for acetone (solid line), ethanol (dashed line) and hexadecane (dot dashed line) for liquid flow in wettable $18 \mu \mathrm{m}$ pores (from Eq. 5). Inset is a top-view image of horizontally-placed patterned tracks with ethanol advancing by capillary action. 


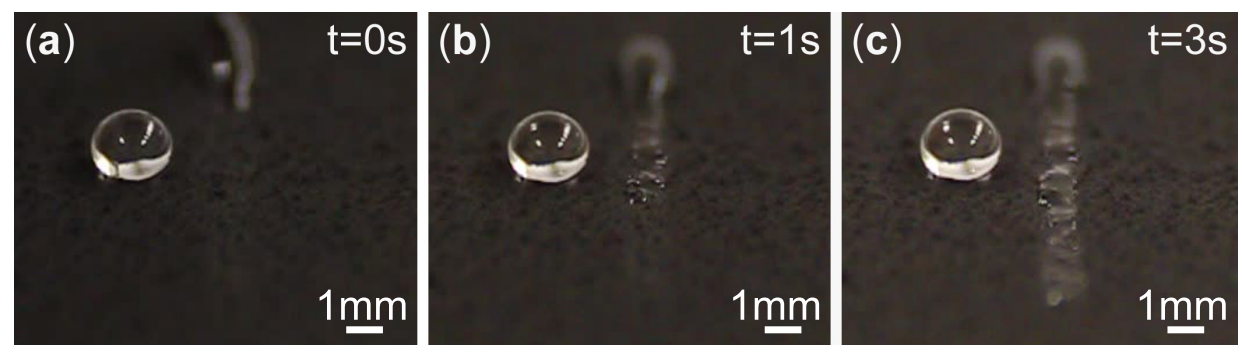

Figure 5: STC line tracks printed with wax on a superoleophobic fluoroacrylic/CNF composite coating. Two identical hexadecane droplets are placed, one outside the wax-coated area (left), and the other (top middle) on the wax line at (a) $t=0 \mathrm{~s}$, (b) $\mathrm{t}=1 \mathrm{~s}$ and (c) $\mathrm{t}=3 \mathrm{~s}$. Note how the fluid advances along the straight track via capillary action. 


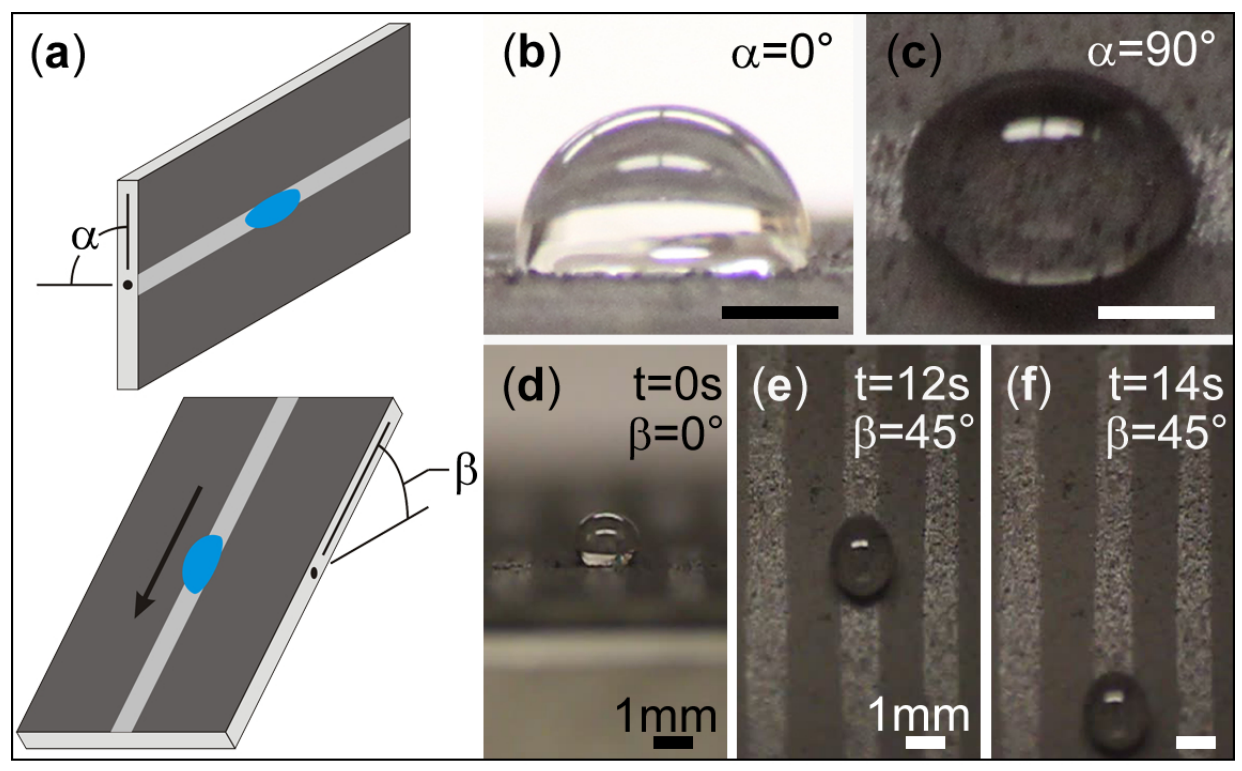

Figure 6: (a) Schematic of anisotropic wetting of water droplets placed in line STC tracks. $\alpha$ and $\beta$ define tilting angles with respect to axes parallel and normal to the track, respectively; droplets remain pinned for $\alpha>0^{\circ}$ and slide for adequate values of $\beta>0^{\circ}$; (b)-(c) demonstrate the confinement of a water droplet (i.e., no mobility) even for $\alpha=90^{\circ}$; (d)-(f) demonstrate droplet sliding action for $\beta=45^{\circ}$ in the time period $t=0$ to $14 \mathrm{~s}$. Inset scale bars are all $1 \mathrm{~mm}$ and the droplet volume is $10 \mu \mathrm{L}$. 


\section{Tables}

Table 1: Apparent advancing CA $\left(\theta_{\mathrm{A}}^{*}\right)$ and receding CA $\left(\theta_{\mathrm{R}}^{*}\right)$ values for liquids of different surface tensions $\left(\gamma_{\mathrm{LG}}\right.$; values obtained from handbook ${ }^{27}$ ) on a superoleophobic PMC-CNF coating (60 wt.\% CNF).

\begin{tabular}{llcl}
\hline \multicolumn{1}{c}{ Liquid } & $\gamma_{\mathrm{LG}}\left(\mathrm{mN} \mathrm{m}^{-1}\right)$ & $\theta_{\mathrm{A}}^{*}\left({ }^{\circ}\right)$ & $\theta_{\mathrm{R}}^{*}\left(^{\circ}\right)$ \\
\hline Water & 71.99 & $160 \pm 3$ & $156 \pm 3$ \\
DMSO & 42.92 & $155 \pm 3$ & $152 \pm 4$ \\
Hexadecane & 27.05 & $157 \pm 2$ & $142 \pm 9$ \\
Ethanol & 21.97 & $144 \pm 3$ & $\approx 0$ \\
Isopropanol & 20.93 & $125 \pm 4$ & $\approx 0$ \\
Hexane & 17.89 & $49 \pm 7$ & $\approx 0$
\end{tabular}


Table 2: Measured and predicted average speeds for fluids of different dynamic viscosity (viscosity values obtained from handbook $\left.{ }^{27}\right)$ to traverse a length $(\Delta x)$ in 1 mm-wide STC tracks.

\begin{tabular}{llcll}
\hline \multicolumn{1}{c}{ Liquid } & $\mu(\mathrm{mPa} \mathrm{s})$ & $\Delta x(\mathrm{~cm})$ & $\frac{\Delta x}{\Delta t}\left(\mathrm{~cm} \mathrm{~s}^{-1}\right)$ & $u$ from Eq. $4\left(\mathrm{~cm} \mathrm{~s}^{-1}\right)$ \\
\hline Acetone & 0.306 & $1.03 \pm 0.01$ & $3.1 \pm 0.5$ & 13.8 \\
Ethanol & 1.074 & $1.08 \pm 0.06$ & $1.2 \pm 0.3$ & 3.68 \\
Hexadecane & 3.032 & $1.08 \pm 0.06$ & $0.7 \pm 0.1$ & 1.61
\end{tabular}

